\title{
The resynchronization therapy in narrow QRS study (RethinQ Study): Methods and protocol design
}

\author{
John F. Beshai • Richard Grimm
}

Published online: 12 February 2008

(C) Springer Science + Business Media, LLC 2007

The publisher regrets that Table 2 from the article, "The resynchronization therapy in narrow QRS study (RethinQ study): Methods and protocol design," which appeared in Journal of Interventional Cardiac Electrophysiology 19:3, pp. 149-155, was incorrectly labeled. The corrected version of Table 2 appears below.

Table 2 Required CRT-D programming

\begin{tabular}{lll}
\hline Parameter & CRT OFF & CRT ON \\
\hline Pacing Mode & VVI (Preferred) DDD & DDD \\
Base Rate & 40 bpm & $40 \mathrm{bpm}$ \\
AV/PV Delay & Must be programmed long enough to allow for a high & As determined during AV/PV optimization \\
& percentage of intrinsic conduction (DDD only) & \\
Interventricular Pace Delay & N/A & As determined during V-V optimization \\
Shortest AV/PV Delay & Must be programmed to 120 ms (DDD only) & 70 ms or longer, as allowed by the optimized AV/PV delay \\
Negative AV/PV Hysteresis & OFF (DDD only) & ON (as allowed by optimized AV/PV delay) \\
\hline
\end{tabular}

The online version of the original article can be found at http://dx.doi. org/10.1007/s10840-007-9156-3.

J. F. Beshai $(\bowtie) \cdot$ R. Grimm

University of Chicago Hospital,

5758 South Maryland Ave., MC 9024,

Chicago, IL 60637, USA

e-mail: jbeshai@medicine.bsd.uchicago.edu 